\title{
Estimating Quantiles of Two Exponential Populations with a Common Location Parameter Using Censored Samples
}

\author{
Adarsha Kumar Jena \\ Department of Mathematics \\ National Institute of Technology Rourkela \\ Rourkela, Odisha-769008, India \\ e-mail: jadarsha@gmail.com \\ Manas Ranjan Tripathy* \\ Department of Mathematics \\ National Institute of Technology Rourkela \\ Rourkela, Odisha-769008, India \\ e-mail:manas@nitrkl.ac.in
}

Received 12 April 2016

Accepted 9 March 2017

\begin{abstract}
Estimation of quantiles of two exponential populations is considered assuming equality of location parameters using censored samples from a decision theoretic point of view. Sufficient conditions for inadmissibility of estimators in certain classes have been derived. Consequently some complete class results as well as estimators dominating the MLE and the UMVUE are obtained. Finally all the proposed estimators have been numerically compared and recommended for use.
\end{abstract}

Keywords: Brewster-Zidek technique; Equivariant estimators; Inadmissibility; Quadratic loss function; Quantile estimation; Relative risk performances; Type-II censoring.

2000 Mathematics Subject Classification: 62F10, 62C15, 62N02.

\section{Introduction}

Suppose we have type-II right censored random samples from two exponential populations with a common location parameter and possibly different scale parameters. More specifically, let $X_{(1)} \leq$ $X_{(2)} \leq \cdots \leq X_{(r)}(2 \leq r \leq m)$ and $Y_{(1)} \leq Y_{(2)} \leq \cdots \leq Y_{(s)}(2 \leq s \leq n)$ be the ordered observations taken from random samples of sizes $m$ and $n$ which follows exponential distributions with a common location parameter $\mu$ and possibly different scale parameters $\sigma_{1}$ and $\sigma_{2}$ respectively. We denote

$\overline{{ }^{*} \text { Corresponding Author }}$

Copyright (C) 2018, the Authors. Published by Atlantis Press.

This is an open access article under the CC BY-NC license (http://creativecommons.org/licenses/by-nc/4.0/). 
$E x\left(\mu, \sigma_{i}\right)$ the exponential population with probability density function

$$
f\left(t, \mu, \sigma_{i}\right)=\frac{1}{\sigma_{i}} \exp \left\{-(t-\mu) / \sigma_{i}\right\}, \quad t>\mu, \sigma_{i}>0,-\infty<\mu<\infty ; i=1,2 .
$$

The problem is to estimate the $p^{\text {th }}$ quantile $\theta=\mu+\eta \sigma_{1}$ of the first population, where $0<\eta=$ $-\log (1-p) ; 0<p<1$. The loss function is taken as

$$
L(d, \underline{\alpha})=\left(\frac{d-\theta}{\sigma_{1}}\right)^{2}
$$

where $d$ is an estimate for estimating the quantile $\theta$ and $\underline{\alpha}=\left(\mu, \sigma_{1}, \sigma_{2}\right)$. We evaluate the performance of an estimator for quantile with the help of the risk function $R(d, \underline{\alpha})=E_{\mu, \sigma_{1}, \sigma_{2}}(L(d, \underline{\alpha}))$.

We note that, for $\eta=0$, the problem reduces to the problem of estimating common location parameter ' $\mu$ ' of two exponential populations using type-II censored samples and has been well investigated by Chiou and Cohen [7] and Tripathy [21]. However, in this article we extend some of their decision theoretic results to the case of estimating quantiles, that is, when $\eta \neq 0$. It should be noted that, exponential distributions have been widely used for analyzing failure time data which arise naturally in various fields of study and we refer to Balakrisnan and Basu [2] and Ahsanulla and Hamedani [1] for some applications of exponential distribution. Exponential quantiles are very much useful in the study of reliability, life testing and survival analysis and some related areas. For some practical application of exponential quantiles we refer to Epstein [10], Epstein and Sobel [11] and Saleh [19].

The problem of estimating parameters of exponential distribution using various types of censored samples has received considerable attention in the recent past by several researchers. Needless to say that the problem under consideration is quite popular as well as important for its real world applications. The applications of this type of models are seen in industry, public health, business, social sciences and related fields that arise naturally in the study of reliability, life testing and survival analysis. Suppose two brands of electrical products have been newly lunched in the market. The life times of the products being random follow exponential distributions. It is also expected that the minimum guarantee time (or equivalently the location parameter $\mu$ ) of both the products are same due to market competition whereas the residual life times (or equivalently the scale parameters) are different. Suppose $m$ and $n$ units respectively, from the two brands have been put for life testing. The experimenter could able to observe only $r(\leq m)$ and $s(\leq n)$ failure times due to certain constraints. On the basis of these sample values one needs to draw the inference on the mean life times or in general the quantile. Most of the commonly used censoring schemes available in the literature are type-I (when number of observations are random and time is fixed), type-II (when number of observations are fixed and time is random), random censoring (both time and number of observations are random) or a mixture of these. For a quick review on estimation of parameters of exponential population using such types of conventional censoring schemes, we refer to Lawless [16] and Johnson et al. [15].

It should be noted that, type-II censoring is a special case of progressive type-II censoring scheme. A lot of attention has been paid in estimating the parameters of an exponential population using progressive type-II censored samples by several authors in the recent past. For some classical as well as decision theoretic results in this direction, we refer to Balakrishnan and Sandhu [4], Chandrasekar et al. [6], and Madi [17]. For some recent updates and detailed review on estimation of parameters of an exponential population using progressive type-II censored samples, one may 
refer to Balakrishnan and Cramer [3]. Estimation of quantiles of an exponential population using doubly censored (both left and right type-II) samples has been considered by Elfessi [8], from a decision theoretic point of view. On the other hand, a little attention has been paid in estimating the parameters, when two or more exponential populations are available. We refer to Chiou and Cohen [7] and Tripathy [21] where the model (1.1) has been previously considered in the literature. Elfessi and Pal [9] considered the estimation of common scale parameter when type-II censored samples are available from several exponential populations.

It is worth mentioning that the model (1.1), we consider in this article reduces to the full sample model when $r=m$ and $s=n$, and that has been well investigated from a classical as well as decision theoretic point of view by several researchers in the recent past. We refer to Ghosh and Razmpour [12], Rukhin [18], Jin and Crouse [14], Sharma and Kumar [20] and Jin and Crouse [13] for some excellent results and review on estimation of common location or/and quantiles of two or more exponential populations when full sample is available.

In this paper our main objective is to estimate the quantiles $\theta=\mu+\eta \sigma_{1}$ of the first population for the model (1.1), using type-II right censored samples. In fact, the results of Sharma and Kumar [20] can be derived as a particular case of our results by choosing $m=r, n=s$ and $m=n$. Basically they have obtained some inadmissibility results for estimating quantiles $\theta$ assuming the sample sizes are equal. They also obtained estimators which dominate the UMVUE in terms of risk values. However, in practice one would be interested to know the percentage of risk improvements approximately. Taking advantages of computational facilities we compare all the proposed estimators numerically. Hence it fills the gap in the literature which is not available. We organize our findings as follows. In Section 2 we discuss the model and present some basic results. In Section 3, we propose a class of estimators which contain the UMVUE of quantiles $\theta$ and obtain estimators dominating the UMVUE. In Section 4, we derive sufficient conditions for improving equivariant estimators and as a consequence some complete class results have been obtained. Most importantly, in Section 5, we carry out a simulation study to numerically compare the risk values as well as the percentage of relative risk improvements of all the proposed estimators which may be useful for practical purposes. Finally we conclude our remarks in Section 6.

\section{Construction of Some Basic Estimators for Quantiles}

In this section, we discuss the model and derive some baseline estimators for the quantile $\theta=$ $\mu+\eta \sigma_{1}$. Specifically we obtain the MLE, a modification to the MLE and the UMVUE for $\theta$.

Let $X_{(1)} \leq X_{(2)} \leq \cdots \leq X_{(r)},(2 \leq r \leq m)$ be the $r$ smallest ordered observations taken from a random sample of size $m$ having probability density function $\operatorname{Ex}\left(\mu, \sigma_{1}\right)$ as given in (1.1). Similarly, let $Y_{(1)} \leq Y_{(2)} \leq \cdots \leq Y_{(s)},(2 \leq s \leq n)$ be the $s$ smallest ordered observations taken from a random sample of size $n$ having probability density function $\operatorname{Ex}\left(\mu, \sigma_{2}\right)$ as given in (1.1). The samples drawn from two populations are assumed to be statistically independent.

For this particular model a sufficient statistic is $\left(U_{1}, U_{2}, Z\right)$, where $Z=\min \left(X_{(1)}, Y_{(1)}\right), U_{1}=$ $\frac{1}{m}\left[\sum_{i=1}^{r} X_{(i)}+(m-r) X_{(r)}\right]$, and $U_{2}=\frac{1}{n}\left[\sum_{j=1}^{s} Y_{(j)}+(n-s) Y_{(s)}\right]$. The joint probability density function of $\underline{U}=\left(U_{1}, U_{2}, Z\right)$ is given by,

$$
f_{\underline{U}}(\underline{u})=K\left(u_{1}-z\right)^{r-1}\left(u_{2}-z\right)^{s-1}\left(\frac{r-1}{u_{1}-z}+\frac{s-1}{u_{2}-z}\right) \exp \left\{\begin{array}{r}
\left.-\frac{m\left(u_{1}-\mu\right)}{\sigma_{1}}-\frac{n\left(u_{2}-\mu\right)}{\sigma_{2}}\right\}, \\
u_{1}>x_{(1)}, u_{2}>y_{(1)}, z>\mu
\end{array}\right.
$$


where $K=\frac{m^{r} n^{s}}{\Gamma s \Gamma r \sigma_{1}^{r} \sigma_{2}^{s}}$, (see Chiou and Cohen [7] and Tripathy [21]). We also note that the random variable $Z$ follows an exponential distribution with location parameter $\mu$ and scale parameter $1 / p$, where $p=m / \sigma_{1}+n / \sigma_{2}$. The MLEs of $\mu, \sigma_{1}$ and $\sigma_{2}$ are obtained by Tripathy [21] and are given by $Z, m\left(U_{1}-Z\right) / r$ (say $\hat{\sigma}_{1 M L}$ ), and $n\left(U_{2}-Z\right) / s$ (say $\hat{\sigma}_{2 M L}$ ) respectively. Using the MLEs of $\mu$ and $\sigma_{1}$, we obtain the MLE of the quantile $\theta=\mu+\eta \sigma_{1}$ as

$$
d_{M L}=Z+\eta \hat{\sigma}_{1 M L}
$$

Further using the modified MLE of the common location parameter $\mu$ (motivated by Ghosh and Razmpour [12]), we propose a modification to the MLE of the quantile $\theta$ as

$$
d_{M M}=Z-\frac{1}{\hat{p}}+\eta \hat{\sigma}_{1 M L}
$$

where $\hat{p}=m / \hat{\sigma}_{1 M L}+n / \hat{\sigma}_{2 M L}$. It is also noted that the sufficient statistics $\left(U_{1}-Z, U_{2}-Z\right)$ and $Z$ are independent and also complete (see Chiou and Cohen [7]). Using the complete and sufficient statistics $\left(U_{1}-Z, U_{2}-Z, Z\right)$, one can easily obtain the UMVUE of the common location parameter $\mu$ as given in Tripathy [21] and derived by Chiou and Cohen [7]. Let us denote $V_{1}=U_{1}-Z$, $V_{2}=U_{2}-Z$. We note that $E\left(V_{1}\right)=\frac{r}{m} \sigma_{1}-p^{-1}$ and $E\left[\left(\frac{V_{2}}{s-1}\right)^{-1}+\left(\frac{V_{1}}{r-1}\right)^{-1}\right]^{-1}=p^{-1}$. Using these results one can easily derive the UMVUE of the quantile $\theta$ as,

$$
d_{M V}=Z+\frac{V_{1} V_{2}(k-1)}{(r-1) V_{2}+(s-1) V_{1}}+k V_{1}, \quad \text { where } k=\eta m / r .
$$

\section{Improving Upon the UMVUE}

In this section we consider a class of estimators which contain the UMVUE for $\theta=\mu+\eta \sigma_{1}$. Using a technique of Brewster and Zidek [5], we obtain an estimator which dominates the UMVUE with respect to the loss function (1.2). Let us consider the class of estimators for estimating the quantile $\theta=\mu+\eta \sigma_{1}$ as $D=\left\{d_{c}: c \in \mathbb{R}\right\}$ where

$$
d_{c}=Z+\frac{V_{1} V_{2}(k-1)}{(r-1) V_{2}+(s-1) V_{1}}+k c V_{1} .
$$

It should be noted that this class contains the UMVUE $d_{M V}$ for $c=1$.

Let us denote $c_{1}=\frac{r}{r+1}, c_{2}=\frac{\eta m(r-2)+1}{\eta m(r-1)}, c_{12}=\max \left\{c_{1}, c_{2}\right\}, c_{3}=\hat{c}\left(\beta^{-}\right)$, where $\beta^{-}=\frac{r+1}{2 m}-$ $\frac{\sqrt{(r+1)^{2}-4 \eta m}}{2 m}$. Further define the constants

$$
\begin{gathered}
c_{*}=\left\{\begin{array}{l}
c_{1}, \text { if } c>c_{1} \\
c_{2}, \text { if } c<c_{2} \\
c, \text { otherwise }
\end{array}\right. \\
c^{*}= \begin{cases}c_{12}, & \text { if } c>c_{12} \\
c_{3}, & \text { if } c<c_{3} \\
c, & \text { otherwise }\end{cases}
\end{gathered}
$$

Theorem 3.1. The class of estimators $d_{c}$ is inadmissible and is improved by $d_{c_{*}}$ if $c_{*} \neq c$ when $\eta>r / m$ and by $d_{c^{*}}$ if $c^{*} \neq c$ when $0<\eta<r / m$. 
Proof. The lines of the proof are very much similar to the arguments used in the proof of Theorem 3.1 of [20], however one needs to use the joint distribution function of $\left(V_{1}, V_{2}\right)$ (as given in Tripathy [21]).

Next we obtain improved estimators for the UMVUE of the quantile $\theta$ by an application of the Theorem 3.1.

Theorem 3.2. Let the loss function be quadratic loss as given in (1.2). The uniformly minimum variance unbiased estimator(UMVUE) $d_{M V}=d_{1}$ for the quantile $\theta=\mu+\eta \sigma_{1}$ is inadmissible and is improved by $d_{c_{1}}$ when $\eta>(r+1) / 2 m$. For $1 / m<\eta<(r+1) / 2 m$ the UMVUE is improved by $d_{c_{2}}$. For $0<\eta<1 / m$ the estimator $d_{M V}$ is admissible and can not be improved.

Remark 3.1. The class of estimators $\left\{d_{c}: c_{2} \leq c \leq c_{1}\right\}$ form an essentially complete class when $\eta>r / m$. The class of estimators $\left\{d_{c}: c_{3} \leq c \leq c_{1}\right\}$ form an essentially complete class when $(r+$ 1) $/ 2 m<\eta<r / m$. The class $\left\{d_{c}: c_{3} \leq c \leq c_{2}\right\}$ form an essentially complete class when $1 / m<$ $\eta<(r+1) / 2 m$. Finally the class $\left\{d_{c}: c_{3}<c<c_{2}\right\}$ is the essentially complete class in the class $D$ when $0<\eta<1 / m$, and can not be improved on by any $d_{c}$.

Remark 3.2. Using the above results it is easy to write the improved estimator which improves upon the UMVUE of the quantile $\theta$. Let $\eta>r / m$ then the estimator which improves upon the UMVUE is obtained as $d_{M V I}=Z+\frac{V_{1} V_{2}(k-1)}{(r-1) V_{2}+(s-1) V_{1}}+\left(\frac{\eta m}{r+1}\right) V_{1}$. It is easy to write the improved estimator for the case $1 / m<\eta<(r+1) / 2 m$. The estimator $d_{1}=d_{M V}$ can not be improved by any $d_{c}$ when $0<\eta<1 / m$. In Section 5, we numerically evaluate the risk functions of these improved estimators and show the percentage of improvement over the UMVUE $d_{M V}$.

\section{An Inadmissibility Result for Affine Equivariant Estimators}

In this section, we introduce the concept of invariance to our problem and obtain a broad class of estimators for quantiles $\theta=\mu+\eta \sigma_{1}$, which are invariant under an affine group of transformations. Further sufficient conditions for improving these estimators are obtained.

Let $G_{A}=\left\{g_{a, b}: g_{a, b}(x)=a x+b, a \in \mathbb{R}^{+}, b \in \mathbb{R}\right\}$ be an affine group of transformations. Under this transformation the problem remain invariant and the form of an affine equivariant estimator for estimating the quantile $\theta$, based on the sufficient statistics $\left(V_{1}, V_{2}, Z\right)$ is obtained as

$$
d\left(Z, V_{1}, V_{2}\right)=Z+V_{1} \phi(V)=d_{\phi}, \text { (say), }
$$

where $\phi: \mathbb{R}^{+} \rightarrow \mathbb{R}$ and $V=V_{2} / V_{1}$. To proceed further, let us define the functions $\phi_{1}$ and $\phi_{2}$ as follows.

$$
\begin{gathered}
\phi_{1}(v)= \begin{cases}\frac{m}{r+s}\left(\eta-\frac{1}{m}\right), & \text { if } 0<v \leq \frac{1}{1-\eta m} \\
\frac{m+n \tau^{+} v}{r+s}\left(\eta-\frac{1}{m+n \tau^{+}}\right), & \text {if } v>\frac{1}{1-\eta m}\end{cases} \\
\phi_{2}(v)=\frac{m}{r+s}\left(\eta-\frac{1}{m}\right),
\end{gathered}
$$


where $\tau^{+}=-\frac{m}{n}+\frac{1}{n} \sqrt{\frac{m(v-1)}{\eta v}}$. For the affine equivariant estimator $d_{\phi}$, we define the functions $\phi_{1}^{*}$ and $\phi_{2}^{*}$ as below.

$$
\begin{gathered}
\phi_{1}^{*}(v)=\left\{\begin{array}{l}
\phi_{1}, \text { if } \phi<\phi_{1} \\
\phi, \text { otherwise }
\end{array}\right. \\
\phi_{2}^{*}(v)=\left\{\begin{array}{l}
\phi_{2}, \text { if } \phi<\phi_{2} \\
\phi, \text { otherwise }
\end{array}\right.
\end{gathered}
$$

Now it is immediate to propose the main result of this section which will help in deriving improved estimators for the quantiles $\theta$ with respect to the quadratic loss function (1.2).

Theorem 4.1. For the affine equivariant estimator $d_{\phi}$ (as given in (4.1)), define the functions $\phi_{1}^{*}$ and $\phi_{2}^{*}$ as given in (4.2) and (4.3) respectively. Let the loss function be the affine invariant loss (1.2).

- The estimator $d_{\phi}$ is inadmissible and is improved by $d_{\phi_{1}^{*}}$, if there exist some values of the parameters $\underline{\alpha}=\left(\mu, \sigma_{1}, \sigma_{2}\right)$ such that, $P\left(d_{\phi} \neq d_{\phi_{1}^{*}}\right)>0$ when $\eta<1 / m$.

- The estimator $d_{\phi}$ is inadmissible and is improved by $d_{\phi_{2}^{*}}$, if there exist some values of the parameters $\underline{\alpha}=\left(\mu, \sigma_{1}, \sigma_{2}\right)$ such that, $P\left(d_{\phi} \neq d_{\phi_{2}^{*}}\right)>0$ when $\eta>1 / m$.

Proof. The proof follows by an application of the Brewster and Zidek (Brewster and Zidek [5]) technique for improving equivariant estimators. The details of the proof has been omitted for brevity.

Remark 4.1. The above theorem basically gives a complete class result. It simply tells that any affine equivariant estimator $d_{\phi}$ of the form (4.1) can be improved if $P\left(\phi<\phi_{1}\right)>0$ (when $\eta<1 / m$ ) or $P\left(\phi<\phi_{2}\right)>0($ when $\eta>1 / m)$.

Remark 4.2. The class of estimators $\left\{d_{\phi}: \phi \geq \phi_{1}\right\}$ for estimating the quantiles $\theta$ form a complete class with respect to the loss function (1.2) when $\eta<1 / m$. The class of estimators $\left\{d_{\phi}: \phi \geq \phi_{2}\right\}$ for estimating the quantiles $\theta$ form a complete class with respect to the loss function (1.2) when $\eta>1 / m$.

It is easy to note that, all the estimators such as the MLE $d_{M L}$, a modification to the MLE $d_{M M}$ (MM) and the UMVUE $d_{M V}$ considered for the quantiles $\theta$ belong to the class $d_{\phi}$ as given in (4.1).

Remark 4.3. Though the estimators $d_{M L}$ and $d_{M M}$ belong to the class $d_{\phi}$ in (4.1), the condition for improving these estimators does not satisfy which has been observed from our simulation study. Hence we are not able to get improved estimator for $d_{M L}$ and $d_{M M}$. However, the UMVUE $d_{M V}$ has been improved by using Theorem 4.1, when $\eta<1 / m$ and denote the improved estimator as $d_{M V A}$. A numerical comparison of this estimator with other estimators has been done using Monte-Carlo simulation method in Section 5.

\section{Simulation Study}

In the previous sections we have proposed various estimators for the quantiles $\theta$ such as the MLE $d_{M L}$, a modification to the MLE (MM) $d_{M M}$ and the UMVUE $d_{M V}$. Further improved estimators $d_{M V I}$ and $d_{M V A}$ dominating the UMVUE have also been derived. However, it should be noted that the analytical comparison of risk values for all these estimators is not possible. Taking the advantages of 
computational facilities, we in this section numerically evaluate the risk values of all these estimators. For this purpose, we have generated 20,000 type-II censored random samples each from two exponential populations having probability density function (1.1) with a common location parameter $\mu$ and different scale parameters $\sigma_{1}, \sigma_{2}$. The loss function is taken as (1.2). We use Monte-Carlo simulation method to compute the simulated risk values of each estimator. The accuracy of simulation has been checked and the standard error is of the order of $10^{-4}$. It can be easily seen that with respect to the loss function (1.2), the risk values of all the estimators are function of $\tau=\sigma_{2} / \sigma_{1}>0$, for fixed sample sizes and fixed $\eta$. The simulation study has been conducted for wide range of the parameters, however for illustrative purpose we report the simulated risk values for some selected choices of parameters. Let us define the percentage of relative risk improvements (RRI) of all the estimators with respect to the MLE as,

$R 1=\left(1-\frac{d_{M M}}{d_{M L}}\right) * 100, R 2=\left(1-\frac{d_{M V}}{d_{M L}}\right) * 100, R 3=\left(1-\frac{d_{M V I}}{d_{M L}}\right) * 100, R 4=\left(1-\frac{d_{M V A}}{d_{M L}}\right) * 100$.

Also we define the percentage of risk improvement of improved estimators over their old estimators,

$$
P 1=\left(1-\frac{d_{M V I}}{d_{M V}}\right) * 100, P 2=\left(1-\frac{d_{M V A}}{d_{M V}}\right) * 100 .
$$

Further we define the censoring factors $(k 1$ and $k 2$ ) for both the populations as the ratio of number of observed samples to the total number of samples. That is for the first population $\mathrm{k} 1 \mathrm{r} / \mathrm{r}$ and for the second population $k 2=s / n$. It can be noticed that the censoring factors $k 1$ and $k 2$ always lie between 0 and 1 . A massive simulation study has been carried out by considering various combinations of sample sizes and $\eta$. However, for illustration purpose, we present (in Table 1) the percentage of relative risk performances as well as percentage of risk improvements for sample sizes $(m, n)=(8,8)$ and for $\eta=1.5, \eta=0.01$. The first column gives the values of $\tau$. Corresponding to one value of $\tau$, there corresponds four values of relative risk performances for an estimator. These four values correspond to $k 1=k 2=0.25,0.50,0.75,1.00$ respectively.

The following conclusions can be drawn from our simulation study as well as the Table 1.

(1) Let $\eta>r / m$ or $(r+1) / 2 m$. The percentage of relative risk values $R 2$ increases with respect to both $\tau$ and $k 1, k 2$, whereas the relative risk value $R 3$ increases for small values of $\tau$ and then starts decreasing after attending maximum somewhere near 1.0. The behavior of $R 1$ is not clear.

(2) Let $\eta<1 / m$. The relative risk improvement $(R 1, R 2, R 4)$ of all the estimators with respect to the MLE $d_{M L}$ increases as the censoring factors $k 1$ and $k 2$ increase for fixed sample sizes. Also $R 1, R 2$ and $R 4$ increases with respect to $\tau$ and attains its maximum somewhere near $\tau=1$, then slowly decrease. Further, as $\tau$ becomes large the risk values of all the estimators converge to some constant value.

(3) The percentage of improvements of $d_{M V I}$ over $d_{M V}(P 1)$ is maximum around $39 \%$ and the percentage of improvements of $d_{M V A}$ over $d_{M V}(P 2)$ is near to $15 \%$. As the censoring factors $k 1$ and $k 2$ increase the percentage of improvement becomes negligible. The maximum improvement is obtained near $\tau=1$.

(4) Consider for small values of $\eta$ that is $\eta<1 / m$. When the values of $\tau$ are close to 0 , the estimator $d_{M M}$ has the maximum percentage of relative risk performance. For moderate values of $\tau(0.25<\tau<3.00)$, the estimator $d_{M V A}$ has the maximum percentage of relative risk improvement and is seen to vary from $30 \%$ to $47 \%$. However, for large values of $\tau$ ( $\geq$ 
3.0) the estimator $d_{M M}$ performs the best and the percentage of relative risk improvement is seen near to $45 \%$.

(5) Consider that $\eta>1.0$ or $\eta>(r+1) / 2 m$. When the values of $\tau$ are close to 0 , and $k 1$ and $k 2$ also close to 0 , the estimator $d_{M M}$ has the maximum percentage of relative risk performance and is seen near to $1 \%$. For moderate to large values of $\tau$, the estimator $d_{M V I}$ has the maximum percentage of relative risk improvement and it is seen near to $36 \%$.

(6) From our simulation study we notice, that the amount of improvement of $d_{M V A}$ over $d_{M V}$ decreases as the values of $\tau$ increases. The improvement is not significant as the values of $k_{1}$ and $k_{2}$ increases for $\eta<1 / \mathrm{m}$. We also observe that the estimator $d_{M V I}$ gives maximum percentage of improvement over $d_{M V}$ for the case $\eta>r / m$.

(7) On the basis of our computational results, we recommend the following. When $\eta$ is small and the values of $\tau$ are close to 0 , we recommend to use $d_{M M}$. For moderate values of $\tau$ we recommend to use $d_{M V A}$ whereas for large values of $\tau$ the estimator $d_{M M}$ is recommended. When $\eta>(r+1) / 2 m$, and for small values of $\tau$, we recommend to use $d_{M M}$ whereas for moderate to large values of $\tau$ we recommend using the estimator $d_{M V I}$.

(8) A similar type of observations have been made for other combinations of $k 1, k 2$ and the sample sizes.

\section{Conclusions and an Example}

In this paper we have considered the estimation of quantiles of two exponential populations assuming that the location parameters are equal using type-II censored samples from a decision theoretic point of view. We have derived some baseline estimators such as the MLE, the modified MLE and the UMVUE for the quantile $\theta$. We also obtained estimators which dominate the UMVUE for $\eta>1 / m$. Further inadmissibility results have been proved for affine equivariant estimators. It should be noted that when the censoring factors $k 1$ and $k 2$ become 1 , the problem reduces to the full sample problem which was earlier studied by several authors including Sharma and Kumar [20]. Though they have obtained improved estimators analytically, it is essential to know the percentage of risk improvement approximately. In this regard our results (Section 5) add one more dimension to their results and may be handy for practical purposes for $k 1=k 2=1$. Also we have obtained the results when the sample sizes are not equal and $k 1=k 2=1$. The present work also extends the results of Tripathy [21] to the case of $\eta \neq 0$ which is new.

Next, we present an example where our model fits well and compute the estimates for the quantile $\theta=\mu+\eta \sigma_{1}$.

Example 6.1. (Simulated Data) Suppose two brands of electronic devices each having 30 units are placed for a life testing experiment. It is known that, the lifetimes (in hours) of each unit follows an exponential distribution with same minimum guarantee time. The experimenter could able to observe only 10 units of failures (in hours) from each brands of devices because of some constraints. The data for both the brands are obtained as Brand 1: 59.69, 60.18, 68.33, 113.78, 155.78, 203.83, 237.86, 243.67, 251.62, 301.49; Brand 2: 37.62, 73.03, 100.54, 103.61, 106.37, 110.72, 119.26, $135.59,169.75,177.03$.

On the basis of above data, we have computed the statistic values as $Z=37.62, V_{1}=219.91$, and $V_{2}=118.18$. Let $\eta=2.0$, then the various estimates for the quantile $\theta=\mu+\eta \sigma_{1}$ have been computed as $d_{M L}=1357.13, d_{M M}=1349.448, d_{M V}=1399.84, d_{M V I}=1279.88$. In this situation, we recommend to use $d_{M V I}$. 
Table 1: Relative risk performances of various estimators for quantile $\theta$ $(m, n)=(8,8)$ with $\mathrm{k} 1=\mathrm{k} 2=0.25,0.50,0.75,1.00$

\begin{tabular}{|c|c|c|c|c|c|c|c|c|}
\hline \multirow[t]{2}{*}{$\tau \downarrow$} & \multicolumn{4}{|c|}{$\eta=1.5$} & \multicolumn{4}{|c|}{$\eta=0.01$} \\
\hline & $R 1$ & $R 2$ & $R 3$ & $P 1$ & $R 1$ & $R 2$ & $R 4$ & $P 2$ \\
\hline \multirow{4}{*}{0.05} & 0.71 & -81.30 & -25.16 & 30.96 & 27.00 & 9.91 & 13.03 & 3.46 \\
\hline & 2.30 & -25.68 & 1.73 & 21.81 & 39.18 & 35.78 & 35.85 & 0.11 \\
\hline & 2.57 & -11.89 & 3.83 & 14.05 & 42.54 & 41.43 & 41.43 & 0.01 \\
\hline & 4.38 & -6.71 & 5.34 & 11.29 & 45.60 & 44.97 & 44.97 & 0.00 \\
\hline \multirow{4}{*}{0.25} & 0.34 & -48.47 & 2.60 & 34.40 & 30.01 & 19.93 & 30.70 & 13.45 \\
\hline & 1.60 & -17.28 & 9.07 & 22.47 & 40.72 & 39.08 & 40.51 & 2.35 \\
\hline & 2.76 & -9.13 & 8.51 & 16.17 & 43.63 & 42.81 & 43.25 & 0.76 \\
\hline & 3.91 & -4.96 & 8.31 & 12.65 & 46.21 & 45.78 & 45.93 & 0.26 \\
\hline \multirow{4}{*}{0.75} & 0.18 & -22.85 & 21.96 & 36.48 & 30.63 & 22.39 & 33.70 & 14.57 \\
\hline & 1.02 & -8.88 & 16.86 & 23.65 & 41.95 & 40.92 & 43.00 & 3.52 \\
\hline & 1.20 & -4.34 & 11.87 & 15.54 & 44.83 & 44.66 & 45.40 & 1.33 \\
\hline & 1.65 & -2.30 & 9.86 & 11.89 & 46.66 & 46.58 & 46.88 & 0.56 \\
\hline \multirow{4}{*}{1.00} & 0.13 & -17.03 & 26.53 & 37.22 & 31.03 & 23.76 & 34.46 & 14.03 \\
\hline & 0.53 & -6.06 & 17.61 & 22.31 & 42.72 & 41.64 & 43.31 & 2.86 \\
\hline & 1.11 & -3.57 & 13.11 & 16.11 & 46.57 & 46.59 & 47.19 & 1.11 \\
\hline & 1.32 & -1.80 & 10.10 & 11.69 & 47.02 & 47.20 & 47.34 & 0.27 \\
\hline \multirow{4}{*}{1.25} & 0.19 & -14.31 & 29.15 & 38.02 & 31.34 & 23.79 & 33.63 & 12.90 \\
\hline & 0.19 & -4.27 & 17.18 & 20.57 & 41.72 & 41.27 & 42.61 & 2.28 \\
\hline & 0.95 & -2.85 & 14.30 & 16.68 & 45.93 & 46.00 & 46.51 & 0.94 \\
\hline & 1.04 & -1.42 & 10.64 & 11.90 & 46.04 & 46.02 & 46.20 & 0.34 \\
\hline \multirow{4}{*}{2.00} & 0.01 & -6.92 & 32.14 & 36.54 & 30.62 & 22.18 & 31.21 & 11.60 \\
\hline & 0.11 & -2.38 & 18.66 & 20.56 & 41.62 & 40.94 & 41.84 & 1.52 \\
\hline & 0.50 & -1.56 & 14.41 & 15.73 & 44.85 & 44.65 & 44.90 & 0.45 \\
\hline & 0.29 & -0.64 & 9.60 & 10.18 & 46.08 & 45.92 & 46.06 & 0.26 \\
\hline \multirow{4}{*}{2.50} & 0.03 & -5.36 & 33.31 & 36.71 & 29.23 & 20.06 & 28.40 & 10.42 \\
\hline & 0.14 & -1.90 & 19.78 & 21.27 & 40.87 & 39.80 & 40.51 & 1.18 \\
\hline & 0.12 & -0.86 & 13.15 & 13.90 & 44.15 & 43.98 & 44.11 & 0.23 \\
\hline & 0.28 & -0.49 & 10.91 & 11.35 & 45.98 & 46.12 & 46.15 & 0.05 \\
\hline \multirow{4}{*}{3.00} & 0.06 & -4.59 & 34.50 & 37.38 & 29.44 & 20.13 & 28.29 & 10.22 \\
\hline & 0.07 & -1.40 & 19.40 & 20.52 & 41.07 & 40.09 & 40.64 & 0.91 \\
\hline & 0.16 & -0.77 & 13.47 & 14.13 & 44.29 & 43.72 & 43.88 & 0.27 \\
\hline & 0.35 & -0.46 & 11.33 & 11.75 & 46.14 & 46.13 & 46.16 & 0.05 \\
\hline \multirow{4}{*}{3.50} & 0.04 & -3.53 & 34.62 & 36.85 & 29.12 & 21.00 & 27.45 & 8.16 \\
\hline & 0.17 & -1.38 & 20.94 & 22.02 & 40.39 & 39.02 & 39.51 & 0.79 \\
\hline & 0.28 & -0.76 & 15.01 & 15.66 & 43.77 & 43.25 & 43.35 & 0.17 \\
\hline & 0.16 & -0.30 & 10.47 & 10.74 & 46.04 & 45.89 & 45.91 & 0.02 \\
\hline \multirow{4}{*}{4.00} & 0.08 & -3.33 & 35.63 & 37.70 & 28.68 & 17.91 & 24.47 & 7.99 \\
\hline & 0.09 & -0.99 & 20.04 & 20.83 & 39.96 & 38.87 & 39.24 & 0.61 \\
\hline & 0.18 & -0.57 & 15.11 & 15.59 & 43.27 & 42.84 & 42.88 & 0.07 \\
\hline & 0.30 & -0.34 & 11.68 & 11.98 & 45.06 & 44.76 & 44.77 & 0.02 \\
\hline \multirow{4}{*}{4.50} & 0.00 & -2.27 & 34.00 & 35.47 & 28.99 & 17.85 & 24.42 & 7.99 \\
\hline & 0.05 & -0.75 & 20.25 & 20.85 & 39.56 & 37.88 & 38.19 & 0.49 \\
\hline & 0.11 & -0.42 & 14.85 & 15.21 & 44.91 & 44.41 & 44.46 & 0.08 \\
\hline & 0.29 & -0.31 & 11.93 & 12.20 & 44.80 & 44.48 & 44.47 & 0.01 \\
\hline \multirow{4}{*}{5.00} & 0.01 & -2.02 & 33.30 & 34.62 & 27.82 & 16.13 & 22.36 & 7.42 \\
\hline & 0.07 & -0.72 & 19.60 & 20.18 & 39.62 & 38.22 & 38.47 & 0.39 \\
\hline & 0.00 & -0.25 & 13.44 & 13.66 & 43.76 & 42.95 & 42.98 & 0.04 \\
\hline & 0.03 & -0.13 & 10.35 & 10.47 & 44.58 & 44.27 & 44.28 & 0.02 \\
\hline
\end{tabular}




\section{Acknowledgments}

The Second author (Manas Ranjan Tripathy) would like to thank Department of Science and Technology (SERB), [SR/FTP/MS-037/2012 dated 4/10/2013] New Delhi, India for providing some financial support.

\section{References}

[1] M. Ahsanulla, and G. G. Hamedani, Exponential Distribution: Theory \& Methods, (Nova Science Publsher Inc. USA. 2011).

[2] N. Balakrishnan, and A. P. Basu, Exponential Distributions: Theory, Methods \& Applications, (Gorden and Breach Publishers, Amsterdam, Netherlands., 1995).

[3] N. Balakrishnan, and E. Cramer, The Art of Progressive Censoring: Applications to Reliability and Quality, (Springer, New York, 2014).

[4] N. Balakrishnan, and R. A. Sandhu, Best linear unbiased and maximum likelihood estimation for exponential distributions under general progressive type-II censored samples, Sankhya: The Indian Journal of Statistics, 58, Series B, Pt.1, (1996), 1-9.

[5] J. F. Brewster, and J. V. Zidek, Improving on equivariant estimators, The Annals of Statistics, 2(1), (1974), 21-38.

[6] B. Chandrasekar, L. T. Alexander, and N. Balakrishnan, Equivariant estimation for parameters of exponential distributions based on type-II progressively censored samples, Communications in StatisticsTheory and Methods, 31(10), (2002), 1675-1686.

[7] W. Chiou, and A. Cohen, Estimating the common location parameter of exponential distributions with censored samples, Naval Research Logistics Quarterly, 31,(1984), 475-482.

[8] A. Elfessi, Estimation of a linear function of the parameters of an exponential distribution from doubly censored samples, Statistics and Probability Letters, 36, (1997), 251-259.

[9] A. Elfessi, A. and N. Pal, On location and scale parameters of exponential distributions with censored observations, Communications in Statistics-Theory and Methods, 20(5-6), (1991), 1579-1592.

[10] B. Epstein, Simple estimates of the parameters of exponential distributions. In Contributions to Order Statistics, A. E. Sarhan and B. G. Greenberg, (Eds.), 361-371, (1962), Wiley, New York.

[11] B. Epstein, and M. Sobel, Some theorems relevant to life testing from an exponential distribution. Annals of Mathematical Statistics, 25, (1954), 373-381.

[12] M. Ghosh, and A. Razmpour, Estimation of the common location parameter of several exponentials, Sankhya: The Indian Journal of Statistics, 46, Series A, Pt. 3, (1984), 383-394.

[13] C. Jin, and H. Crouse, An identity for exponential distributions with the common location parameter and its applications, Communications in Statistics-Theory and Methods, 27(2), (1998a), 409-422.

[14] C. Jin, and H. Crouse, A note on the common location parameter of several exponential populations, Communications in Statistics-Theory and Methods, 27(11), (1998b), 2777-2789.

[15] N. L. Johnson, S. Kotz, and N. Balakrishnan, Continuous Univariate Distributions, Vol-1, John Wiley \& Sons, INC, New York, (2004).

[16] J. F. Lawless, Statistical Models and Methods for Lifetime Data, 2nd Edition, Wiley, New York, (2003)

[17] M. T. Madi, A note on the equivariant estimation of an exponential scale using progressively censored data, Journal of Statistical Planning and Inference, 140, (2010), 1437-1440.

[18] A. Rukhin, Admissibility and minimaxity results in the estimation problem of exponential quantiles, The Annals of Statistics, 14(1), (1986), 220-237.

[19] A. K. Md. E. Saleh, Estimating quantiles of exponential distributions. In:Statistics and Related Topics, Csorgo, M., Dawson, D., Rao, J. N. K., Saleh, A. K. Md. E. (Eds.), . North Holland, Amsterdam, (1981), 279-283.

[20] D. Sharma, and S. Kumar, Estimating quantiles of exponential populations, Statistics and Decisions, 12, (1994), 343-352.

[21] M. R. Tripathy, Equivariant estimation of common location parameter of two exponential populations using censored samples, Hacettepe Journal of Mathematics and Statistics, 45(4), (2016), 1307-1320. 